\title{
Analysis of the Autonomic Regulation in a Case of Facioscapulohumeral Muscular Dystrophy after Ken Ware Treatment
}

\author{
Ken Ware', Elio Conte ${ }^{2,3}$, Riccardo Marvulli' ${ }^{2,4}$, Giancarlo Ianieri'2,4, Marisa Megna ${ }^{2,4}$, \\ Enrico Pierangeli², Sergio Conte' ${ }^{2}$, Leonardo Mendolicchioº ${ }^{2}$, Flavia Pellegrino ${ }^{2}$ \\ ${ }^{1}$ The International Neuro Physics Functional Performance Institute, Energy Circuit, Robina, Australia \\ ${ }^{2}$ School of Advanced International Studies on Applied Theoretical and Non Linear Methodologies of Physics, \\ Bari, Italy \\ ${ }^{3}$ Department of Basic Sciences, Neuroscience and Sense Organs, University Aldo Moro, Bari, Italy \\ ${ }^{4}$ Department of Physical Medicine and Rehabilitation, University Aldo Moro, Bari, Italy \\ Email: elio.conte@fastwebnet.it
}

Received 23 April 2015; accepted 26 May 2015; published 29 May 2015

Copyright (C) 2015 by authors and Scientific Research Publishing Inc.

This work is licensed under the Creative Commons Attribution International License (CC BY).

http://creativecommons.org/licenses/by/4.0/

(c) (7) Open Access

\section{Abstract}

This is a study on autonomic neuroscience. In a previous paper in [1], we studied a subject affected from facioscapulohumeral muscular dystrophy before and after Ken Ware treatment (NPT). Using the non linear methodology of the Generalized Mutual Information (GMI) analysis of Sensory Motor Rhythm, we produced detailed results evidencing that the mentioned NPT treatment involved a net improvement of the patient under his subjective psychological condition, and in particular, under the neurological and sensory motor profile. We quantified with accuracy the improvement that the subject realized during such treatment. Of course, previous studies of several authors have evidenced that muscular dystrophies are strongly linked to a profound ANS disfunction. Therefore, the aim of the present study was to analyze the ANS of the subject before and after the treatment. We performed analysis in time as well as in frequency domain and by using non linear methods. The basic result of the paper was that, according to our analysis, the subjects started with a serious ANS disfunction before the NPT treatment and that a net improvement was obtained after this therapy. All the examined parameters resulted strongly altered before the treatment and all they returned in the normal range after the NPT.

\section{Keywords}

Autonomic Neuroscience, HRV Analysis, Ken Ware (NPT) Treatment, Facioscapulohumeral Muscular Dystrophy, ANS Dysfunction 


\section{Introduction}

When speaking about Heart Rate Variability (HRV), we have to start considering such basic foundations. Baroreceptor activity is received from the Nucleus Tractus Solitarius (NTS) that provides to transmit on one hand the signal to Nucleus Ambigous/Dorsal Motor Nucleus (NA/DMN) integrated by the hypothalamus action which generates finally parasympathetic heart rhythm modulation and on the other hand the signal to Rostral Ventrolateral Medulla oblongata (RVLM) that integrates hypothalamus contribution generating orthosympathetic heart rhythm modulation. A complete scheme of the complex arrangement may be found in [2]

On the basis of this scheme, the HRV may be considered as an analysis that on one hand gives important information about the fitness of heart to respond to the autonomic nervous system (ANS) modulation, and on the other hand (and it is here the important feature relating the present paper) gives direct indication on the manner in which some central brain structures of dominant relevance are explicating their basic function when inducing heart rhythm modulation. In this sense, we may say that HRV dynamics is the "mirror image" of the psychophysiological mechanisms involved in some central brain regions. By this way, we conclude that HRV studies represent currently a non invasive marker not only to explore the cardiovascular system but also to indirectly investigate some important neurological involved functions.

To support this thesis, we have to quote here a recent neuroimaging study [3]. This study was conducted by Julian F. Thayer, Fredrik Åhs, Mats Fredrikson, John J. Sollers and Tor D. Wager who evidenced the existing main relationship between Hear Rate (HR) and regional brain blood flow. These authors indicated in detail that the more significant involved brain regions were represented from the amygdala and from the ventromedial prefrontal cortex. In detail, these authors identified the medial pfc (MPFC) (right pregenual cingulate), the MPFC (right subgenual cingulated) and the SLEA (left sublenticular extended amigdala/ventral striatum).

Our conclusion is that such results evidence the importance of the HRV studies in exploring cognitive as well as emotive functions in clinical conditions of subjects affected from psychological disorders as well as psychiatric pathologies. This is not a restriction of course since, as we will attempt to evidence in the present paper, the heart rate variability analysis may be considered of importance also in the study of subjects affected from neuromuscular pathologies.

In 2003, we started to analyze HRV in different experimental conditions. And in the course of several years of studies we have also introduced novel methods [4]-[6] of analysis in order to obtain valuable quantifications of the VLF, LF, and HF bands where in particular the quantification obtained in the LF band is related mainly to the modulation due to the orthosympathetic activity while the HF band relates more directly parasympathetic activity and respiration.

The aim of the present study is to evaluate HRV and thus ANS disfunction in a subject affected from Facioscapulohumeral Muscular Dystrophy and to estimate its improvement after that the subject has been submitted to a treatment that we have called NPT that is due to Ken Ware and that we have explained in detail recently elsewhere [1].

We may reassume briefly the reason to investigate here the ANS disfunction before and after Ken Ware treatment. As previously outlined in [1], Duchenne (DMD) and Becker (BMD) muscular dystrophies are disorders due to complete or partial loss of dystrophin protein. The presence of persistent sinus tachycardia, atrial and ventricular arrhythmias, sweating and chills has suggested an ANS involvement in these disorders. Yotsukura et $a l$. first described an impairment of ANS in 55 DMD, characterized by an increase in sympathetic activity and a reduction in parasympathetic output [7] [8]. ANS impairment was confirmed also by studies that were developed by Lanza et al. [9] and by Inoue et al. [10] [11]. In a study developed from Vita in 2001 [12], 20 BMD patients were investigated with a battery of six cardiovascular autonomic tests and power spectral analysis of HRV. Although 11 patients revealed abnormal findings at some cardiovascular tests, none of them had a definite autonomic damage, as indicated by two or more abnormal tests. This clinical observation suggested therefore that autonomic involvement did not represent a major finding in BMD. In 2006, the prognostic value of HRV for sudden death was evaluated in a population of 30 BMD patients and dilated cardiomyopathy compared with a control group of normal subjects [13]. The important result was that an increment of sympathetic tone in BMD was observed, characterized by a lower HRV in the frequency and time domains and a higher mean heart rate than in the control group.

When distinguishing the case of the myotonic dystrophy (DM) in the myotonic dystrophy type 1 (DM1) and type 2 (DM2) with different clinical phenotypes, we find that in such conditions we have indication of ANS in- 
volvement such as heart rhythm conduction disturbances, orthostatic hypotension, disorders of sweating, abnormal gut movements, and dysfunction of genitourinary apparatus as studied in particular from [14]. Very important results may be found in [10] [15]-[26].

Facio Scapulo Humeral Muscular Dystrophy (FSHD) is the third most frequent form of muscular dystrophy. In [27], HRV was evaluated in 55 FSHD patients and evidenced the presence of autonomic modifications, characterized by a slight increase in sympathetic activity and a progressive decrease in parasympathetic output, which became more evident with the progression of the disease.

Emery-Dreifuss Muscular Dystrophy (EDMD) is genetically heterogeneous since it may be caused by a mutation in the STA gene encoding emerin (X-linked inheritance) or as AD trait determined by mutations in the LMNA gene encodinglamin A/C. Scintigraphic studies [28] evidenced a diffuse and severe decrease in accumulation of MIBG in the heart, suggesting an abnormality in the cardiac sympathetic nerve terminals in the patient.

Miyoshi muscular dystrophy (MMD) is caused by mutation in the gene encoding the protein dysferlin. In 1994, Tomoda et al. [29] performed accurate autonomic studies in 2 MMD affected girls, including laser doppler flowmetry, study of the component analysis of the cardiographic R-R interval and sympathetic skin response (SSR). They demonstrated marked abnormalities such as sensitive vasoconstrictive response, a suppressed peak of low frequency components, and an absence of SSR, compared with healthy controls.

We retain to have delineated a sufficient picture of the importance of evaluation of HRV and ANS disfunction in muscular dystrophy. The present paper is a case report. We give the results on ANS renormalization in a severe case of muscular dystrophy after the treatment that is introduced by Ware and discussed and analyzed in detail by us elsewhere [1].

\section{Materials, Methods and the NPT Treatment}

As it was outlined by us in detail in [1] the case is of a subject 60-year-old-male suffering for more than 30 years from the rare form of Muscular Dystrophy, Facioscapulohumeral Muscular Dystrophy (FSHD). (FSHD) is a genetic muscle disorder in which the muscles of the face, shoulder blades and upper arms are among the most affected. EEG, ECG, EMG were all recorded simultaneously using BioRadio 150 technology. EEG was recorded in channel 8 with electrodes placed at C3-F3 of the left hemisphere. ECG was recorded in Channel 7 with electrodes placed at V1 and V2. An appropriate software by BioPac sytem enabled us to obtain the relative R-R intervals for HRV analysis. The patient was recorded for 5 minutes at rest before therapy in order to perform quantitative estimations before and after the treatment. The exercise equipment used is specialized exercise equipment supplied to the International NeuroPhysics Functional Performance Institute by "Synergy Fitness (http://synergyfitness.com.au/gallery/15/Synergy 4 Pin_Loaded) and was described in detail min [1]. In substance this treatment that we have called the Ken Ware NeuroPhysics Treatment (NPT) does not involve physical manipulation of the treated individual. Usually early phase equipment is the leg press (which may be substituted with the seated leg curl), lateral pull down, and pectoral exercise, the pec dec. Each exercise machine loaded with very light weight, mostly representative of the equipment's minimum lifting loads. Most movements have only two degrees of freedom ( $d f$ ) (up-down or forward-back). Only one machine used in the early phase of treatment has $4 d f$, namely the pec dec. The initial treatment in our case, involved four models of variable resistance exercise equipment, which were selected in consideration to the level of ability of the patient. No stretching or warm-up exercises are permitted to preserve a patient's system normal resting state, innocent of perturbations until the (NPT) starts. The initial assessment session(s) evidences the patient's chronic system condition and how it interacts with and evaluates its environments in general. The specific activities and equipment used at the beginning of treatment are expanded after initial sessions' effects area assessed and systematically modulated as necessary, through the unique applications and qualities of NPT. In the next treatment phase, additional exercises that are slightly more complex to perform, but with still very lightly weighted, are added to the patients program, again depending on ability or disability. Some of these pieces of equipment have many $(d f)$; the handles are attached to cables that can move in any direction. The patients are at this time (re) introduced to socalled "cardio" exercises (this nuisance term promoted by the exercise industry un-holistically implies the cardiovascular system is separate, requiring its own form of exercise). In the reinforcement and maintenance phase of the program, additional equipment with prescribed repetitions and weights is introduced. Individuals in trea-t ment perform the following activities with trained coaching support). In conclusion, we may identify six phases of the treatment. 
The first phase is devoted the subject to learn to relax physically, mentally, and emotionally.

The second phase is finalized to adjust position for proper contact points and symmetrical balance on equipment.

The third phase is devoted to maintain up right posture.

In the fourth and fifth phases, the subject closes the eyes and performs ultra-slow movements.

Finally, the sixth phase is devoted to form and to operationalize the intention to distribute effort and energy throughout the body (pec dec). For further detail the reader is addressed to ref. [1].

\section{The Results}

As previously outlined, we performed HRV analysis starting with an ECG recorded at $960 \mathrm{~Hz}$. We used linear and non linear methods. As previously discussed, studies in literature have evidenced relationship between lowfrequency power (Power Spectral Density, PSD) of heart rate variability and muscle sympathetic nerve activity (MSNA). We studied HRV before, during the treatment, and days after the treatment.

By using Fourier transform (FFT or DFT), one obtains balancing inspection of the ANS modulation in HRV in the three VLF, LF and HF bands. The VLF Band relates hormonal activity, thermoregulation and possibly the rennin-angiotensin system, the LF band relates mainly the orthosympathetic activity and, finally, the HF band relates instead the parasympathetic activity in the frequency domain.

Let us explore the DFT results evaluating the ANS condition of the subject at rest before starting the treatment. We identify this at rest section as Lat Pull1bt. Let us give a look at the tachogram and to the DFT spectrum as they are reported in Figure 1.

Figure 1 evidences that we are in presence of a subject with very exaggerated beat-to-beat variations and presence of an arrhythmia to be classified. In the PSD spectrum peaks are represented in the three bands all having abnormal amplitudes in the three bands respectively.

Let us give a look at the results of the quantitative analysis. The bpm (beats per minute) of the subject resulted to be $68.7 \mathrm{bpm}$ where we consider a normal range included between 59 and $96 \mathrm{bpm}$. Soon after we examined the variance as it results from the reconstructed tachogram starting with the given R-R time series. Usually, we attribute a great relevance to such index since it informs us directly on the variability induced on the heart rhythm by the ANS modulation and on the availability of the sinus node to respond to ANS activity. The value resulted of 135.5 mseconds and we adopt as normal range the interval 20 - 60 mseconds in the age range of 50 - 70 years old. This result indicated an exaggerated variability in heart rhythm induced from some kind of arrhythmia. The toal Power (PSD) resulted to be $18,397 \mathrm{msec}^{2} / \mathrm{Hz}$ with natural logarithmic value of 9.81 . Usually, we consider as normal range the interval $6.0-8.1$. Therefore, we concluded for the presence of an exaggerated modulation total value exceedingly the normal prefixed value. The VLF band gave as result the value of $3207 \mathrm{msec}^{2} / \mathrm{Hz}$ with $\mathrm{Ln}$ value of 8.07 and the normal range is considered usually to be between 4.5 and 6.4 . The obtained value resulted exaggerated exceeding the established normal range.

The LF band gave us a value of $7565 \mathrm{msec}^{2} / \mathrm{Hz}$ with an Ln value of 8.93 while the normal range is included between 5.0 and 6.9. Finally the HF band gave us a result of $6956 \mathrm{msec}^{2} / \mathrm{Hz}$ with an Ln value of 8.84 in the normal range included between 4.5 and 6.7 .

Also all the linear time indexes resulted exaggerated out of the normal range.
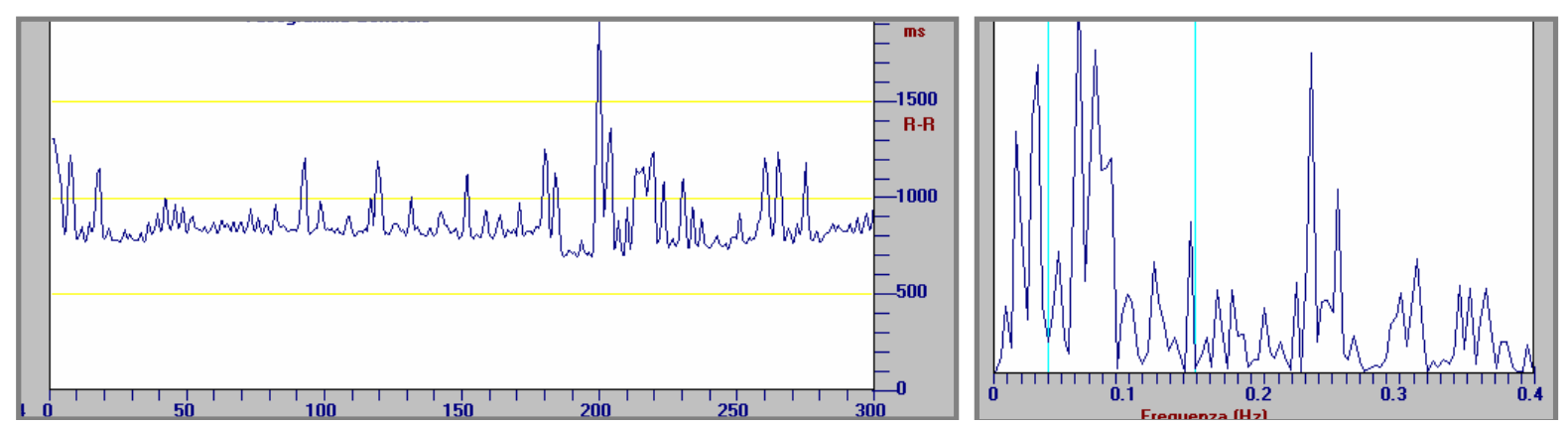

Figure 1. Tachogram obtained after ECG recording, PSD Spectrum $\left(\mathrm{msec}^{2} / \mathrm{Hz}\right)$ with three delineated bands, VLF, LF and HF. 
They are reported in Figure 2.

The context previously delineated configures a net ANS disfunction but in order to deep the problem we need to add further investigations. This was the reason to apply to the data the CZF method that we introduced in literature as explained in detail in [4]-[6]. The CZF method enables us to develop a detailed analysis of the hearth rhythm variability in the frequency domain evaluating in the detail the variability induced from orthosympathetic and parasympathetic modulating activities respectively. The results are reported in the Figure 3 and Figure 4.

The case of the CZF application with absolute value quantifies variability between each pair of subsequent value taking the result in mseconds while the case without absolute value quantifies such variability but accounting if the subsequent time beat value is greater or les of the previous one. The first estimates the value of the beat-to beat difference. The second approach estimates in addition if such interval increases (acceleration of beat to beat interval) or decreases.

In red we have time dynamics and on the right we Fourier analysis of the heart rhythm variability.

The important result that we obtain with this kind of analysis is that it evidences that in the LF band we have a strong reduction of variability and this is to say that we have a strong dominance of sympathetic activity while the HF band that of course characterizes the ANS modulation of the parasympathetic activity remains in the normal range. Therefore, the results appear to be unquestionable. The ANS heart rhythm modulation of this

\begin{tabular}{|c|c|c|c|c|c|}
\hline \multicolumn{6}{|c|}{ All Data } \\
\hline & RRI & HR & & RRI & HR \\
\hline Maximum: & $896.66 \mathrm{~ms}$ & $180.00 \mathrm{bpm}$ & InHRV: & 10.6457 & 7.6497 \\
\hline Minimum: & $333.33 \mathrm{~ms}$ & $66.92 \mathrm{bpm}$ & Coef. of Variance: & $33.5693 \%$ & $46.6346 \%$ \\
\hline Max/Min: & 26900 & 26900 & Variance: & $42010.5849 \mathrm{~ms}^{*} \mathrm{~ms}$ & $2100.1200 \mathrm{bpm}{ }^{*} \mathrm{bpm}$ \\
\hline Range: & $563.33 \mathrm{~ms}$ & $113.09 \mathrm{bpm}$ & Std Dev. (SDNN): & $204.9648 \mathrm{~ms}$ & $45.8271 \mathrm{bpm}$ \\
\hline Mean of NN: & $610.57 \mathrm{~ms}$ & $98.27 \mathrm{bpm}$ & Std Err. (SE): & $11.5853 \mathrm{~ms}$ & $2.5903 \mathrm{bpm}$ \\
\hline Mean of dNN [MSD]: & $48.9419 \mathrm{~ms}$ & $17.0533 \mathrm{bpm}$ & SDSD: & $7901207 \mathrm{~ms}$ & $27.4312 \mathrm{bpm}$ \\
\hline Median: & $769.99 \mathrm{~ms}$ & $77.92 \mathrm{bpm}$ & RMSSD: & $78.9939 \mathrm{~ms}$ & $27.3872 \mathrm{bpm}$ \\
\hline 95\% Conf. Interval: & $22.7072 \mathrm{~ms}$ & $5.0770 \mathrm{bpm}$ & NN50 Count: & 114 & 114 \\
\hline $99 \%$ Conf. Interval: & $29.8414 \mathrm{~ms}$ & $6.6721 \mathrm{bpm}$ & pNN50: & $36.4217 \%$ & $36.4217 \%$ \\
\hline
\end{tabular}

Figure 2. Linear time indexes of the subject at rest.

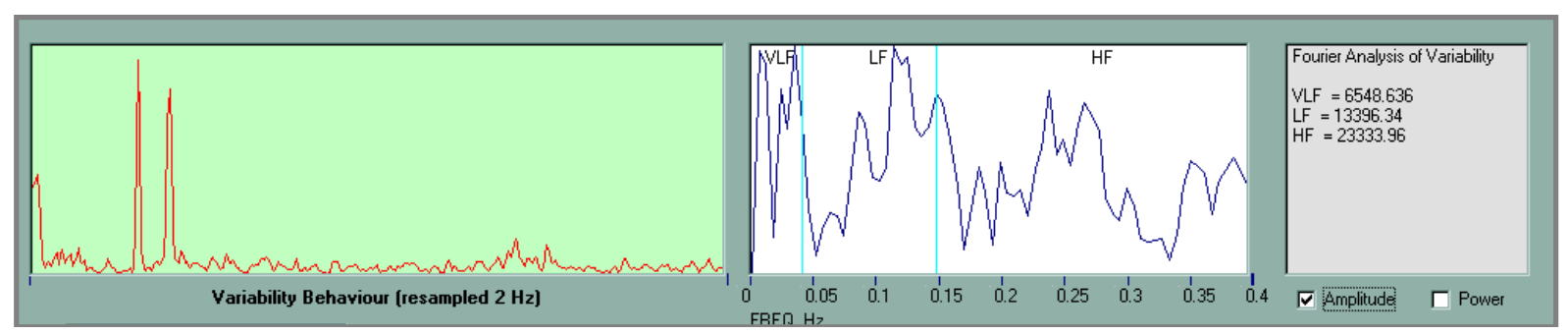

Figure 3. Time series of heart rhythm variability and values of variability ( $\mathrm{msec} / \mathrm{Hz})$ in the case with absolute value (for details see [4]-[6]). The CZF method.

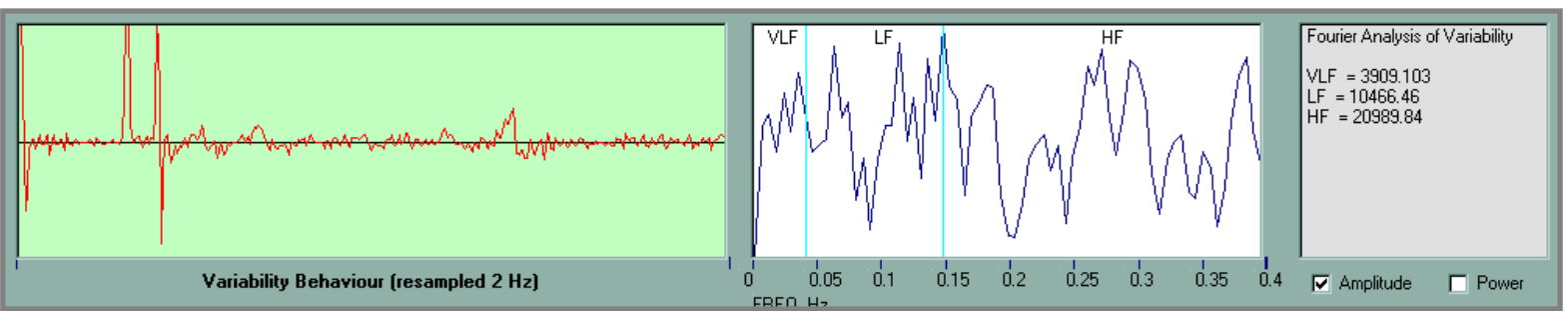

Figure 4. Time series of heart rhythm variability and values of variability $(\mathrm{msec} / \mathrm{Hz})$ in the case without absolute value (for details see [4]-[6]). The CZF method. 
subject is affected from an hyper orthosympathetic activation. This is in agreement with the results of other authors as we discussed in detail in the introduction.

At this point it is instructive to examine also the histograms computed one time for the heart rate (bpm) and one time for the R-R intervals. We give the results in Figure 5 and Figure 6 respectively.

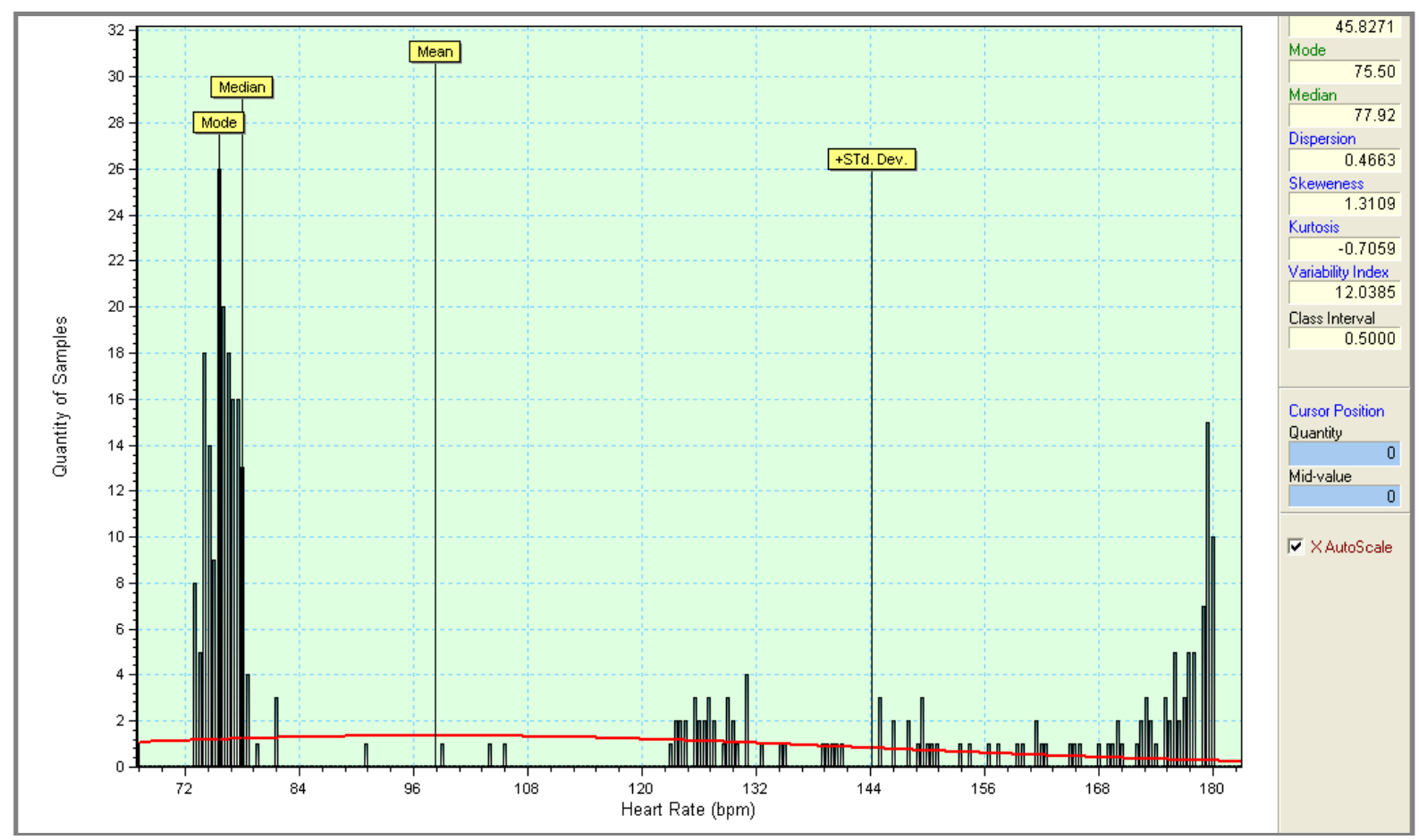

Figure 5. Histograms of HR for the subject at rest.

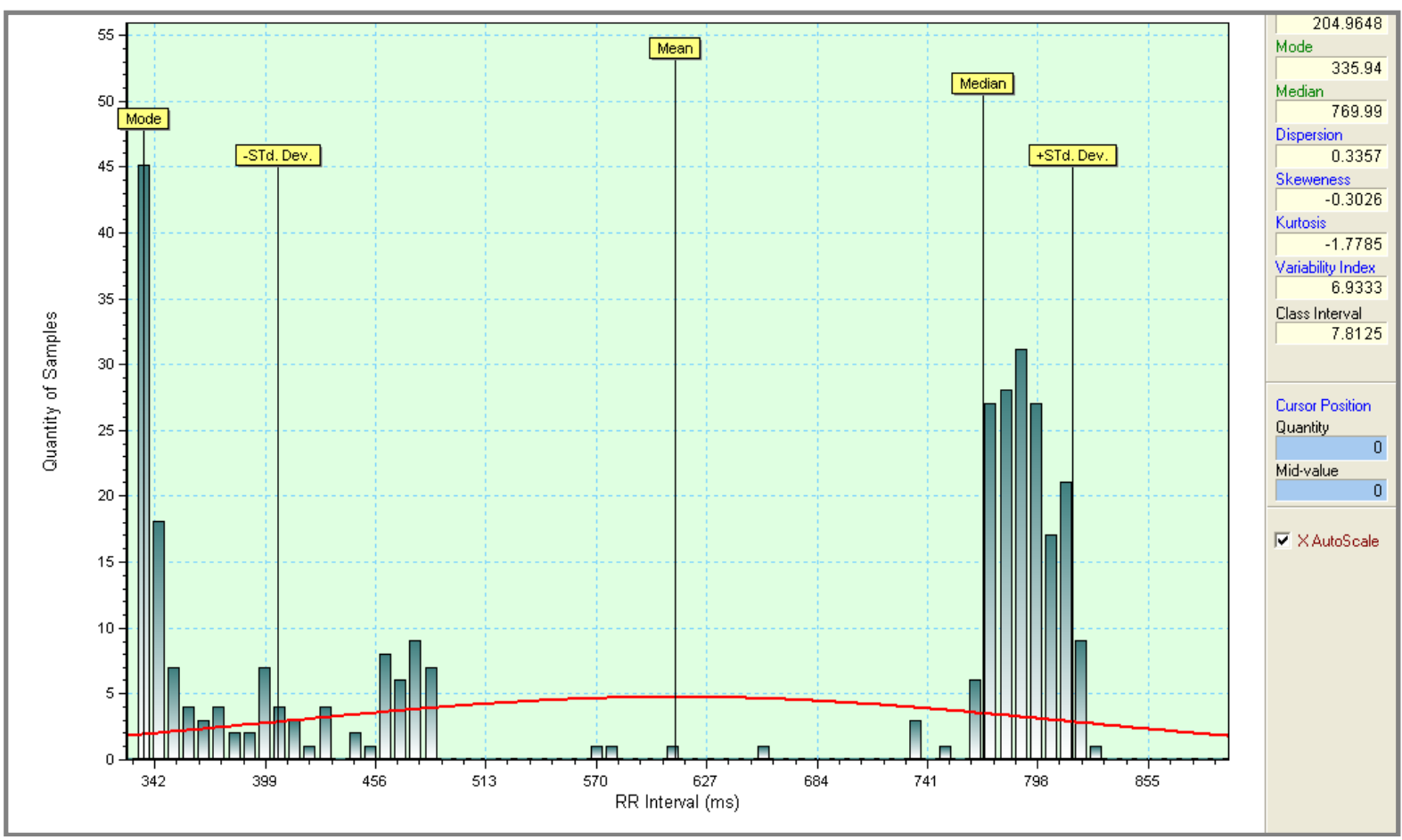

Figure 6. Histograms of R-R intervals for the subject at rest. 
The analysis of HR histograms also reveals a situation of the subjects affected by a profound arrhythmia with HR values violating a normal Gaussian distribution. Mean, Median and Mode are out of the normal requirement in a normal distribution and we observe substantially a distribution peaked about three central values, a peak in HR about 76 (bpm), another peak about 128 (bpm) and a final distribution about $180(\mathrm{bpm})$ The same evaluation is obtained obviously when we consider the distribution of the R-R values as reported in Figure 6. We have substantially a plurimodal distribution that is always indicative of a serious happening arrhythmia in the examined subject.

To complete the analysis we may apply the Poincaré method. We give the results in Figure 7.

This is a well know representation in bi-dimensional phase space. Also its interpretation, obtained on the basis of the distribution of the points and of the estimated values for SD1, SD2, ellipse area and centroid, is well known. As confirmation also of the results previously obtained, this is a clear case of atrial fibrillation.

In detail, the examination of the Poincare plot given in Figure 7 in conjunction with the results previously given, enables us to classify the kind of arrhythmia of the subject. In the plot $R R_{n} / R_{n+1}$ we identify two locations out of the ellipse, one located in the range $310-496 \mathrm{~ms}$ and the other in the range 748 - $868 \mathrm{~ms}$. The Sd1 value is reduced to about $51 \mathrm{~ms}$ or 9.17 n.u. (orthosympathetic activity) against the $284.63 \mathrm{~ms}$ or 46.66 n.u. for SD2 with SD1/SD2 = 0.1966 strongly distant from a condition of acceptable balance parasympathetic/orhosympathetic modulation. The Ellipse Area is strongly out of a normal range. This picture enables us to conclude that the subject is suffering of a persistent arrhythmia to be classified as atrial fibrillation.

We may now conclude our examination by using non linear analysis, estimating in detail Approximate Entropy (ApEn), Sample Entropy (SnEp), and the Detrended Fluctuation Analysis (DFA). They are given in Table 1.

The results are given in Table 1. It is confirmed the profound condition of great cardiovascular risk of the subject and his serious ANS disfunction. This condition is clearly evidenced when considering the values of ApEn and SampEn that are so distant from a valuable value near to 1. Fortunately long as well as short range correlations as estimated by the DFA still remain in the range of an acceptable value.

We may now pass to examine the question of the treatment of the subject connecting in this manner the great

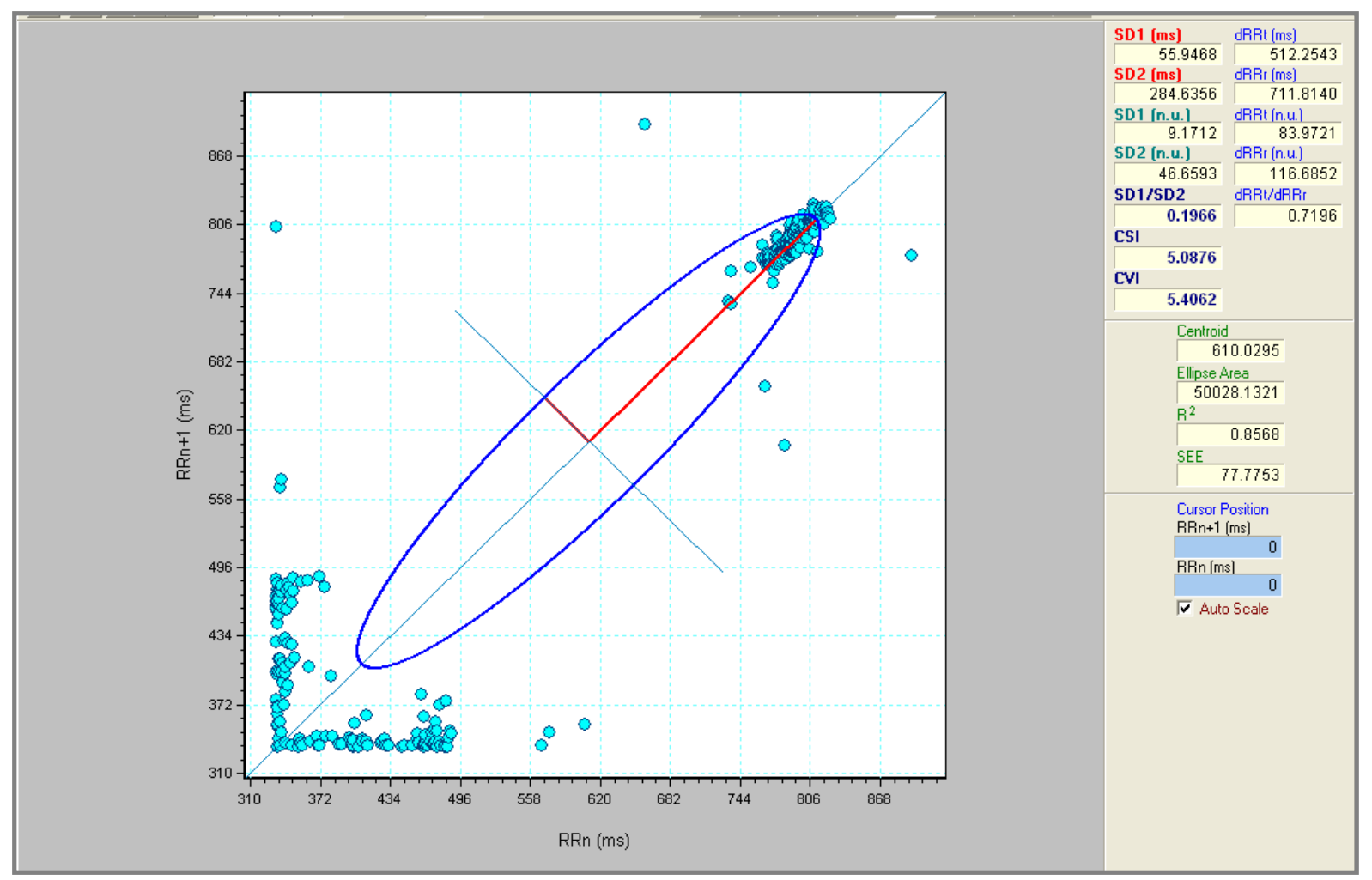

Figure 7. Poincaré Plot relating the subject at rest. 
Table 1. Estimation of approximate entropy, of sample entropy, and detrended fluctuation analysis in the subject at rest.

\begin{tabular}{cc}
\hline Approximate Entropy (ApEn) & 0.194 \\
Sample Entropy (SampEn) & 0.127 \\
Detrended fluctuation (DFA): a1 & 0.963 \\
Detrended fluctuation (DFA): a2 & 1.536 \\
\hline
\end{tabular}

interest from the general neurological interest. After the Ken Ware treatment, the previously mentioned indexes change as it follows.

The Fourier transform.

The subject has now $72 \mathrm{bpm}$. The most important thing is that the variability is now of 30 mseconds that is in the normal range. The total power (PSD) is now $902 \mathrm{msec}^{2} / \mathrm{Hz}(\mathrm{Ln}=6.8)$ (against the value at rest of 18,397 $\left.\mathrm{msec}^{2} / \mathrm{Hz}\right)$ and is now in the normal range. The VLF band gives a value of $464 \mathrm{msec}^{2} / \mathrm{Hz}(\mathrm{Ln}=6.13)$ and is in the normal range, the LF band is $313 \mathrm{msec}^{2} / \mathrm{Hz}(\mathrm{Ln}=5.74)$ and is now in the normal range, the HF band is 94 $(\ln =4.54)$ and still is in the normal range. In Figure 8, we report the tachogram and the Fourier spectrum and both the behaviours re-enter now in the normal regime.

Let us start examining the results obtained by Fourier Transformation and given in Figure 7.

Looking at the Figure 8, we may appreciate under the neurological profile the valuable improvement induced from the NPT treatment. The ANS modulation has totally recovered.

Also the values of the time linear indexes now result in their normal range as shown in Figure 9.

Also the values of the CZF method result to be totally in the normal range. It is very interesting to examine the histograms and the Poicaré Plots. The histograms are given in Figure 10 and Figure 11, HR and R-R intervals respectively.

The histograms follow now the expected normal distribution that we observe in a subject with missing disfunctions.

Evaluating all such results, we may conclude that the ANS has totally recovered its modulating function.

Let us look at the Poicaré Plot in Figure 12.

Looking at Figure 12, we observe that the subject has totally recovered the characteristic distribution at cigar of normal subjects also if still remain some extra-distribution episodes and the form of the cigar is not so largely extended as in a health normal subject. Sd1 and SD2 values are however in the normal range of values as well as the values of the ellipse area and centroid. In detail, the SD1 and the SD2 values, relating the ANS modulation, enter now, as said, in the range of normal values with a correct balancing parasympathetic-orthosympathetic action, that in fact results to be $\mathrm{Sd} 1 / \mathrm{SD} 2=0.848$ (against the value of 0.193 at rest before of the treatment) with $\mathrm{SD} 1=43.94 \mathrm{~ms}$ and $5.22 \mathrm{n}$.u. and $\mathrm{SD} 2=51.79 \mathrm{~ms}$ and 6.15 n.u.

Finally, from a neurological point of view, the most interesting result is given by using non linear indexes. Let us report the results relating the Approximate Entropy (ApEn), the Sample Entropy (SnEp) and the Detrended Fluctuation Analysis (DFA).They are given in Table 2.

All the results are now in the normal range.

\section{Conclusions}

This is a case report of ANS analysis in a subject affected by muscular dystrophy. The HRV was analyzed by using linear and non linear analysis. The analysis performed initially on the subject at rest and before of the treatment by the linear time indexes and traditional FFT resulted out of the range.

The use of the CZF method, histograms and Poincare plot evidenced that the subject started with a strong ortho-sympathetic against parasympathetic unbalance and atrial fibrillation. After the treatment, the patient evidenced that all the standard HRV indexes re-entered in the normal range of values in consideration of his starting arrhythmia, and that in particular balancing orthosympathetic-para-sympathetic correct modulation was recovered.

Under the neurological profile, we have to outline here the profound correlation existing in autonomic regulation and muscular dystrophy. In particular, the use of standard methodologies in HRV analysis, as well as the use of the CZF method and of non linear estimations, enables us to estimate and to correlate the ANS dysfunction with the severity of the muscular pathology. In fact, the results on EMG analysis, published elsewhere [1] and 

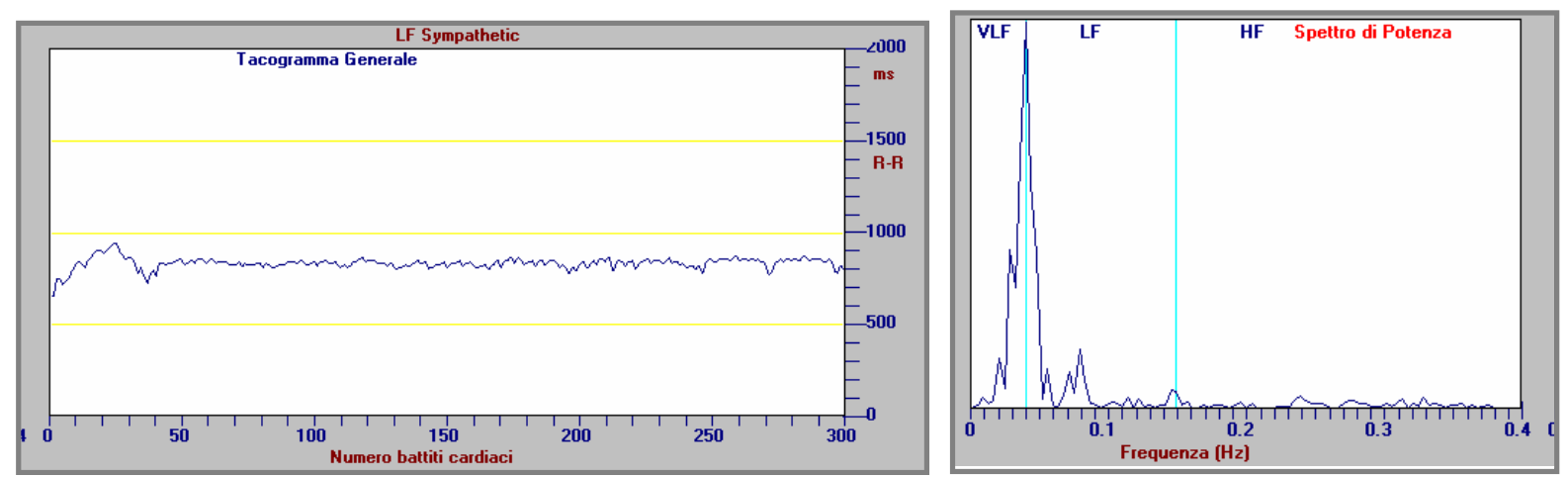

Figure 8. Tachogram obtained after ECG recording after the treatment PSD Spectrum $\left(\mathrm{msec}^{2} / \mathrm{Hz}\right)$ with three delineated bands, VLF, LF and HF.

\begin{tabular}{|c|c|c|c|c|c|}
\hline \multicolumn{6}{|c|}{ All Data } \\
\hline & RRI & HR & & RRI & HR \\
\hline Maximum: & $1243.00 \mathrm{~ms}$ & $135.44 \mathrm{bpm}$ & InHRV: & 7.8592 & 3.7469 \\
\hline Minimum: & $443.00 \mathrm{~ms}$ & $48.27 \mathrm{bpm}$ & Coef. of Variance: & $6.0451 \%$ & $9.1343 \%$ \\
\hline Max/Min: & 2.8059 & 2.8059 & Variance: & $2589.5140 \mathrm{~ms}^{*} \mathrm{~ms}$ & 42.3878 bpm"bpm \\
\hline Range: & $800.00 \mathrm{~ms}$ & $87.17 \mathrm{bpm}$ & Std Dev. (SDNN): & $50.8873 \mathrm{~ms}$ & $6.5106 \mathrm{bpm}$ \\
\hline Mean of NN: & $841.79 \mathrm{~ms}$ & $71.28 \mathrm{bpm}$ & Std Err. (SE): & $3.0575 \mathrm{~ms}$ & $0.3912 \mathrm{bpm}$ \\
\hline Mean of dNN [MSD]: & $16.5000 \mathrm{~ms}$ & $1.6264 \mathrm{bpm}$ & SDSD: & $62.1444 \mathrm{~ms}$ & $7.0684 \mathrm{bpm}$ \\
\hline Median: & $843.00 \mathrm{~ms}$ & $71.17 \mathrm{bpm}$ & RMSSD: & $62.0461 \mathrm{~ms}$ & $7.0591 \mathrm{bpm}$ \\
\hline $95 \%$ Conf. Interval: & $5.9927 \mathrm{~ms}$ & $0.7667 \mathrm{bpm}$ & NN50 Count: & 6 & 6 \\
\hline 99\% Conf. Interval: & $7.8756 \mathrm{~ms}$ & $1.0076 \mathrm{bpm}$ & pNN50: & $2.1661 \%$ & $2.1661 \%$ \\
\hline
\end{tabular}

Figure 9. Values of Linear time indexes in HRV analysis after the treatment.

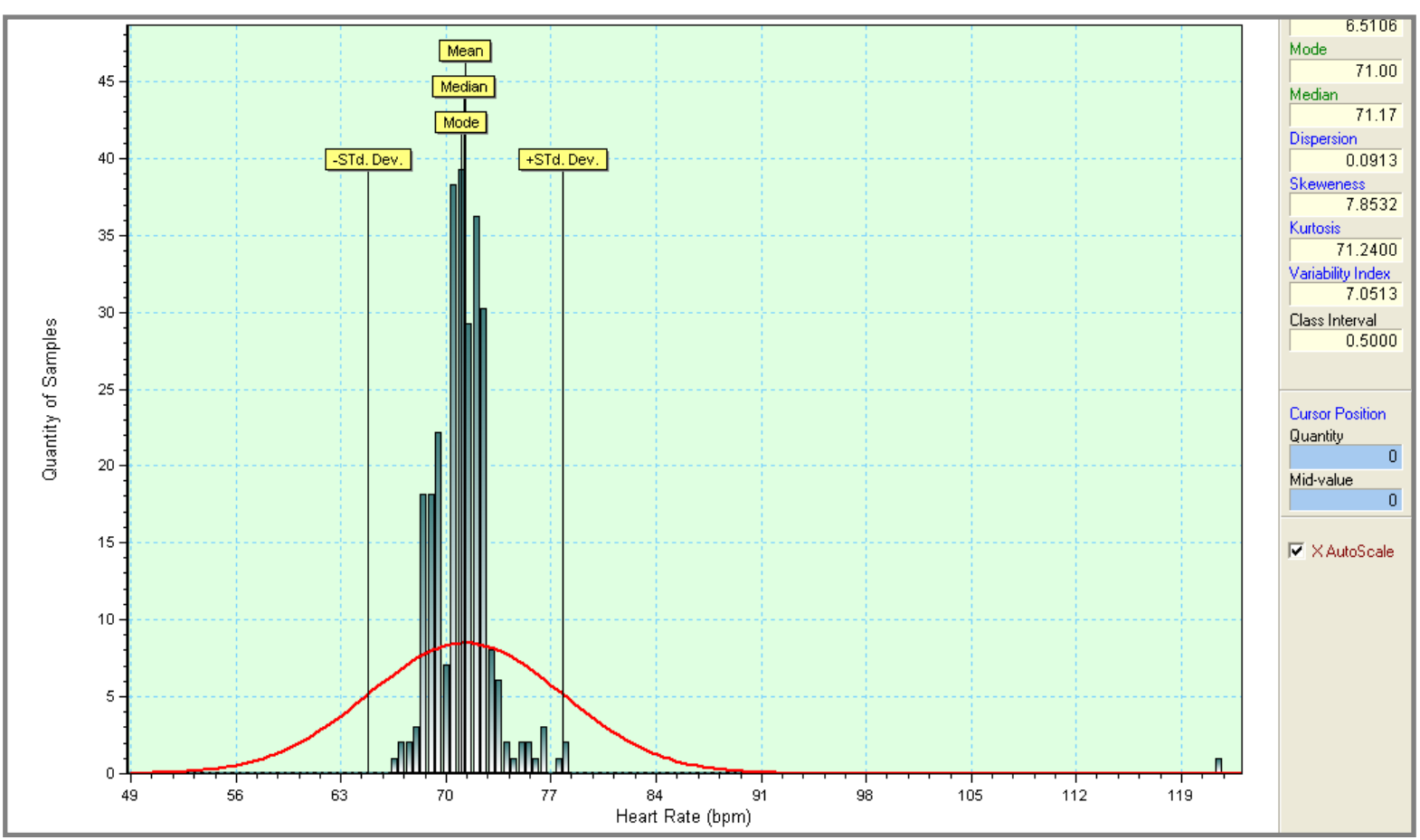

Figure 10. HR-Histogram distribution of the HR values after NPT treatment. 


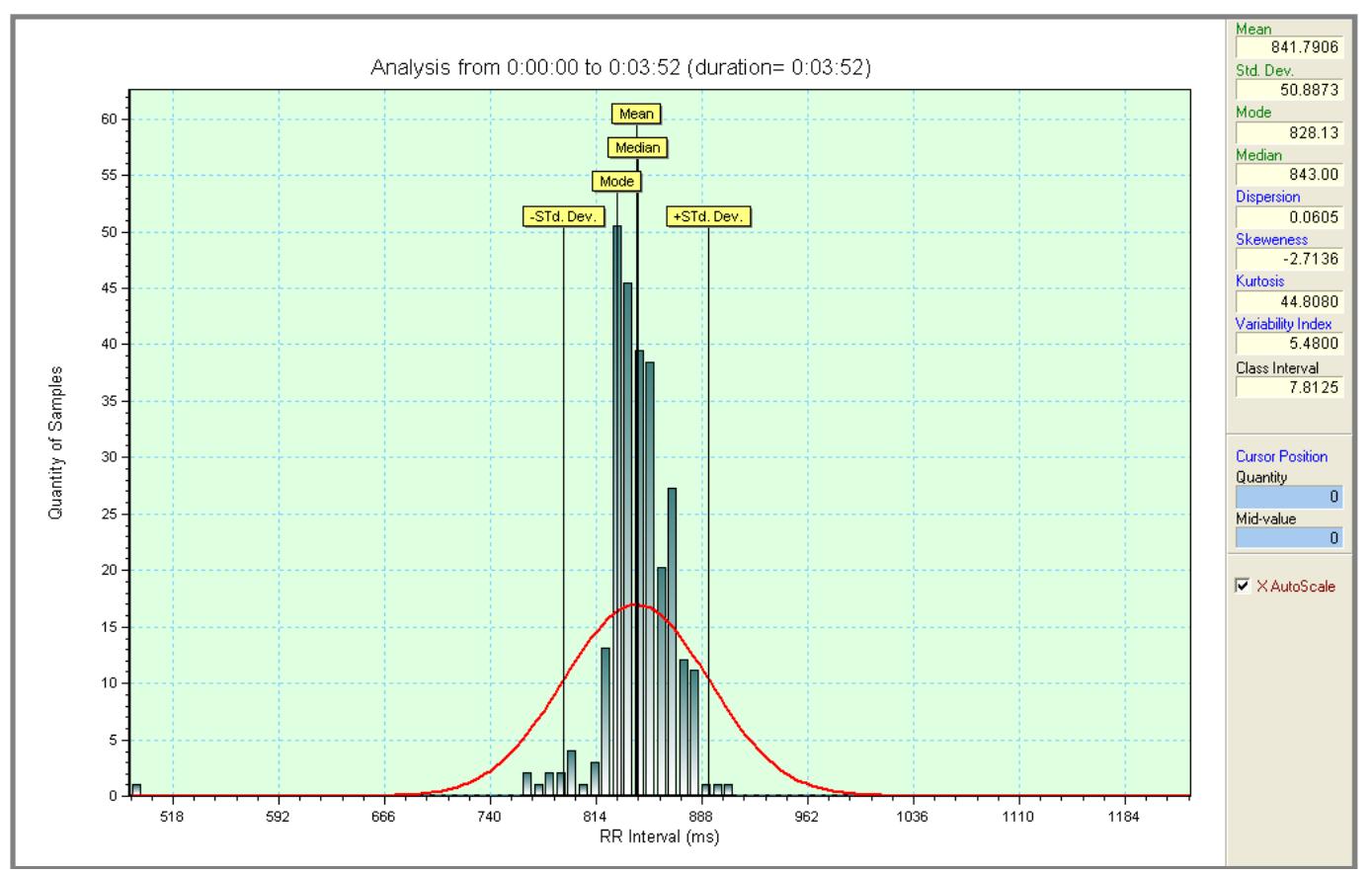

Figure 11. R-R Histogram distribution of the RR values after NPT treatment.

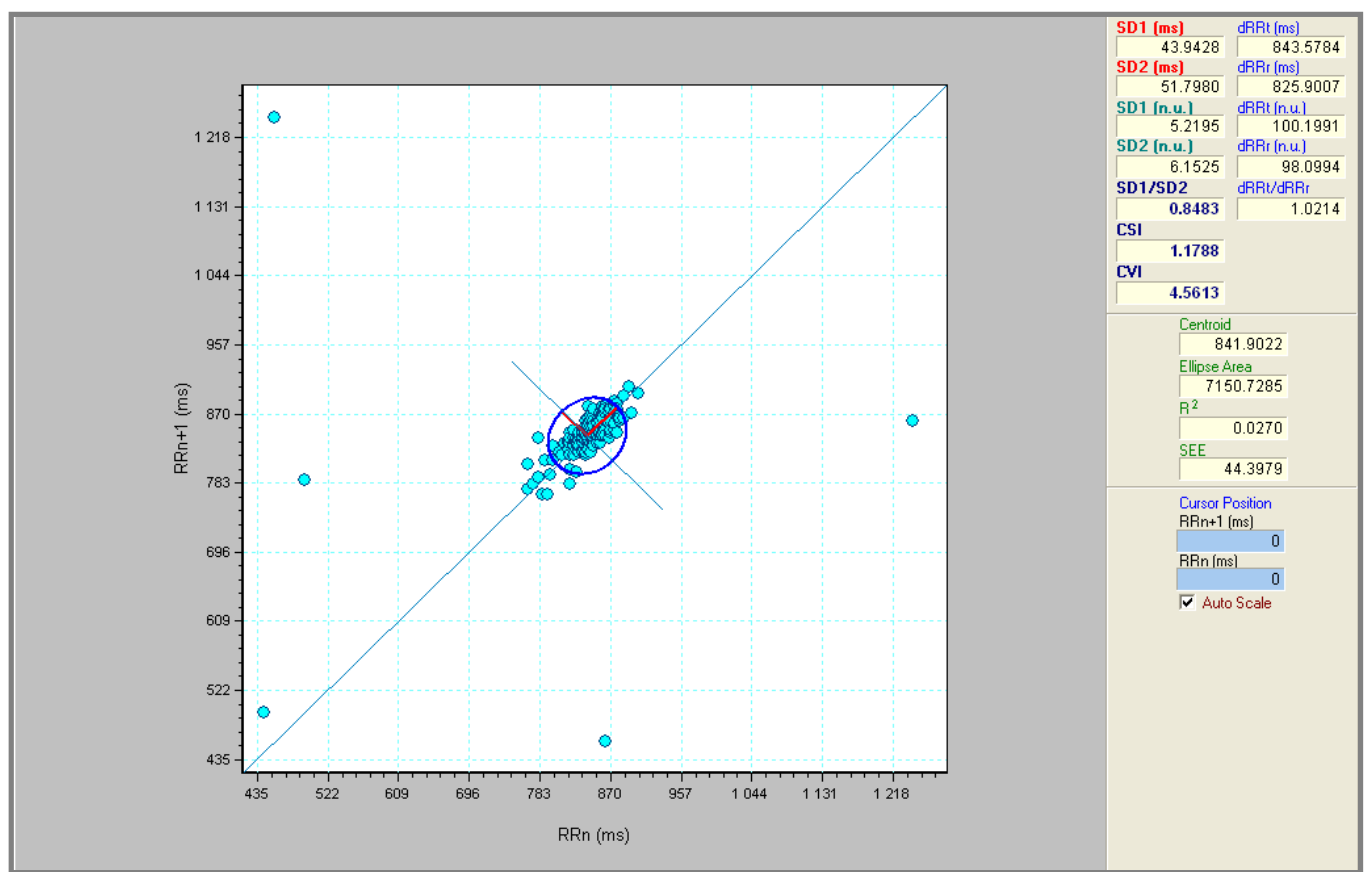

Figure 12. Poincaré Plot of the subject after NPT treatment.

Table 2. Estimation of approximate entropy, of sample entropy, and detrended fluctuation analysis in the subject after the NPT treatment.

$\begin{array}{cc}\text { Approximate Entropy (ApEn) } & 0.887 \\ \text { Sample Entropy (SampEn) } & 0.913 \\ \text { Detrended fluctuation (DFA): a1 } & 0.802 \\ \text { Detrended fluctuation (DFA): a2 } & 0.973\end{array}$


relating this subject, evidenced that ha had a so serious initial compromission that of course resulted to improve under the profile of the clinical and pathophysiological conditions after the treatment.

\section{References}

[1] Ware, K., Conte, E., Marvulli, R., Ianieri, G., Megna, M., Pierangeli, E., Conte, S., Mendolicchio, L. and Pellegrino, F. (2015) Case Report: Generalized Mutual Information (GMI) Analysis of Sensory Motor Rhythm in a Subject Affected by Facioscapulohumeral Muscular Dystrophy after Ken Ware Treatment. World Journal of Neuroscience, 5, 67-81. http://dx.doi.org/10.4236/wjns.2015.52008

[2] Conte, E. (2008) Clinical Electrophysiology: Principles and Methods of Analysis, Edises.

[3] Thayer, J.F., Åhs, F., Fredrikson, M., Sollers, J.J. and Wager, T.D. (2012) A Meta-Analysis of Heart Rate Variability and Neuroimaging Studies: Implications for Heart Rate Variability as a Marker of Stress and Health. Neuroscience and Biobehavioral Reviews, 36, 747-756. http://dx.doi.org/10.1016/j.neubiorev.2011.11.009

[4] Conte, E., Giroldini, W., Laterza, V., Conte, S., Pieralice, M., Casciaro, F., De Masi De Luca, G., Giuliano, A.F.M., Mendolicchio, L. and Todarello, O. (2014) Experimental Results on a New Method for Analysis of Heart Rate Variability. World Journal of Cardiovascular Diseases, 4, 385-389. http://dx.doi.org/10.4236/wjcd.2014.48048

[5] Conte, E., Federici, A. and Zbilut, J.P. (2009) A New Method Based on Fractal Variance Function for Analysis and Quantification of Sympathetic and Vagal Activity in Variability of R-R Time Series in ECG Signals. Chaos, Solitons and Fractals, 41, 1416-1426. http://dx.doi.org/10.1016/j.chaos.2008.05.025

[6] Conte, E. (2014) A New Method for Analysis of Heart Rate Variability, Asymmetry and BRS. Chaos and Complexity Letters, 8, 8-15.

[7] Yotsukura, M., Fujii, K., Katayama, A., Tomono, Y., Ando, H., Sakata, K., et al. (1998) Nine-Year Follow-Up Study of Heart Rate Variability in Patients with Duchenne-Type Progressive Muscular Dystrophy. American Heart Journal, 136, 289-296. http://dx.doi.org/10.1053/hj.1998.v136.89737

[8] Yotsukura, M., Sasaki, K., Kachi, E., Sasaki, A., Ishihara,T. and Ishikawa, K. (1995) Circadian Rhythm and Variability of Heart Rate in Duchenne-Type Progressive Muscular Dystrophy. The American Journal of Cardiology, 76, 947-951. http://dx.doi.org/10.1016/S0002-9149(99)80267-7

[9] Lanza, G.A., Dello Russo, A., Giglio, V., De Luca, L., Messano, L., Santini, C., et al. (2001) Impairment of Cardiac Autonomic Function in Patients with Duchenne Muscular Dystrophy: Relationship to Myocardial and Respiratory function. American Heart Journal, 141, 808-812. http://dx.doi.org/10.1067/mhj.2001.114804

[10] Inoue, K., Ogata, H., Matsui, M., Hayano, J., Miyake, S., Kumashiro, M., et al. (1995) Assessment of Autonomic Function in Myotonic Dystrophy by Spectral Analysis of Heart-Rate Variability. Journal of the Autonomic Nervous System, 55, 131-134. http://dx.doi.org/10.1016/0165-1838(95)00040-5

[11] Inoue, M., Mori, K., Hayabuchi, Y., Tatara, K. and Kagami, S. (2009) Autonomic Function in Patients with Duchenne Muscular Dystrophy. Pediatrics International, 51, 33-40. http://dx.doi.org/10.1111/j.1442-200X.2008.02656.X

[12] Vita, G., Di Leo, R., De Gregorio, C., Papalia, A., Rodolico, C., Coglitore, S., et al. (2001) Cardiovascular Autonomic Control in Becker Muscular Dystrophy. Journal of the Neurological Sciences, 186, 45-49. http://dx.doi.org/10.1016/S0022-510X(01)00500-7

[13] Ammendola, E., Russo, V., Politano, L., Santangelo, L. and Calabrò, R. (2006) Is Heart Rate Variability a Valid Parameter to Predict Sudden Death in Patients with Becker's Muscular Dystrophy? Heart, 92, 1686-1987. http://dx.doi.org/10.1136/hrt.2005.082909

[14] Aminoff, M.J., Beckley, D.J. and McIlroy, M.B. (1985) Autonomic Function in Myotonic Dystrophy. Archives of Neurology, 42, 16-20. http://dx.doi.org/10.1001/archneur.1985.04060010018007

[15] Melacini, P., Villanova, C., Menegazzo, E., Novelli, G., Danieli, G., Rizzoli, G., et al. (1995) Correlation between Cardiac Involvement and CTG Trinucleotide Repeat Length in Myotonic Dystrophy. Journal of the American College of Cardiology, 25, 239-245. http://dx.doi.org/10.1016/0735-1097(94)00351-P

[16] Ewing, D.J. and Clarke, B.F. (1982) Diagnosis and Management of Diabetic Autonomic Neuropathy. BMJ, 285, $916-$ 918. http://dx.doi.org/10.1136/bmj.285.6346.916

[17] van Ravenswaaij-Arts, C.M., Kollée, L.A., Hopman, J.C., Stoelinga, G.B. and van Geijn, H.P. (1993) Heart Rate Variability. Annals of Internal Medicine, 118, 436-447. http://dx.doi.org/10.7326/0003-4819-118-6-199303150-00008

[18] Olofsson, B.O., Niklasson, U., Forsberg, H., Bjerle, P., Andersson, S. and Henriksson, A. (1990) Assessment of Autonomic Nerve Function in Myotonic Dystrophy. Journal of the Autonomic Nervous System, 1990, 187-192. http://dx.doi.org/10.1016/0165-1838(90)90144-8

[19] den Heijer, J.C., van Dijk, J.G., Bollen, W.L., Bos, J.E. and Wintzen, A.R. (1991) Assessment of Autonomic Function in Myotonic Dystrophy. Journal of Neurology, Neurosurgery \& Psychiatry, 54, 531-534. 
http://dx.doi.org/10.1136/jnnp.54.6.531

[20] Pierangeli, G., Lugaresi, A., Contin, M., Martinelli, P., Montagna, P., Parchi, P., et al. (1992) Autonomic Nervous System Function in Myotonic Dystrophy. Italian Journal of Neurological Sciences, 13, 589-592. http://dx.doi.org/10.1007/BF02233402

[21] Di Leo, R., Rodolico, C., De Gregorio, C., Recupero, A., Coglitore, S., Annesi, G., et al. (2004) Cardiovascular Autonomic Control in Myotonic Dystrophy Type 1: A Correlative Study with Clinical and Genetic Data. Neuromuscular Disorders, 14, 136-141. http://dx.doi.org/10.1016/j.nmd.2003.11.002

[22] Rakoèevi'c-Stojanovi'c, V., Milovanovi'c, B., Ivi'c, N., Ille, T., Marjanovi'c, I., Stevi'c, Z., et al. (2007) Cardiac Autonomic Nervous System in Patients with Myotonic Dystrophy Type 1. Acta Myologica, 26, 112-114.

[23] Politano, L., Palladino, A., Nigro, G., Scutifero, M. and Cozza, V. (2008) Usefulness of Heart Rate Variability as a Predictor of Sudden Cardiac Death in Muscular Dystrophies. Acta Myologica, 27, 114-122.

[24] Hardin, B.A., Lowe, M.R., Bhakta, D. and Groh, W.J. (2003) Heart Rate Variability Declines with Increasing Age and CTG Repeat Length in Patients with Myotonic Dystrophy Type 1. Annals of Noninvasive Electrocardiology, 8, 227321. http://dx.doi.org/10.1046/j.1542-474X.2003.08310.x

[25] Magrì, D., Piccirillo, G., Bucci, E., Pignatelli, G., Cauti, F.M., Morino, S., et al. (2012) Increased Temporal Dispersion of Myocardial Repolarization in Myotonic Dystrophy Type 1: Beyond the Cardiac Conduction System. International Journal of Cardiology, 156, 259-264. http://dx.doi.org/10.1016/j.ijcard.2010.10.132

[26] Fregonezi, G., Araujo, T., Dourado, M.E., Ferezini, J., Silva, E. and Resqueti, V. (2012) Heart Rate Variability in Myotonic Dystrophy Type 1 Patients. Arquivos Brasileiros de Cardiologia, 98, 353-361. http://dx.doi.org/10.1590/S0066-782X2012005000021

[27] Della Marca, G., Frusciante, R., Scatena, M., Dittoni, S., Testani, E., Vollono, C., et al. (2010) Heart Rate Variability in Facioscapulohumeral Muscular Dystrophy. Functional Neurology, 25, 211-216.

[28] Fujiita, T., Shimizu, M., Kaku, B., Kanaya, H., Horita, Y., Uno, Y., et al. (2005) Abnormal Sympathetic Innervation of the Heart in a Patient with Emery-Dreifuss Muscular Dystrophy. Annals of Nuclear Medicine, 19, 411-414. http://dx.doi.org/10.1007/BF03027407

[29] Tomoda, A., Zhao, J.E., Ohtani, Y., Miike, T., Uchino, M. and Higuchi, I. (1994) Two Patients with Distal Muscular Dystrophy and Autonomic Nerve Dysfunction. Brain \& Development, 16, 65-70. http://dx.doi.org/10.1016/0387-7604(94)90116-3 